\title{
CONFIGURACIÓN DE LA PALA DE KAYAK, LAS MEDIDAS ANTROPOMÉTRICAS Y VARIABLES CINEMÁTICAS*
}

\author{
DR. ARTURO DÍAZ SUÁREZ \\ Doctor en ciencias de la educación - Universidad de Murcia \\ Profesor titular de la Facultad de Ciencias del Deporte - Universidad de Murcia (España) \\ E-mail:ardiaz@um.es \\ DR. JESÚS GARCÍA-PALLARÉS \\ Ldo. en Ciencias de la Actividad Física y el Deporte UCAM (España) \\ E-mail: jgpallares@um.es
}

\begin{abstract}
RESUMEN
Se analizaron las correlaciones entre la configuración de la pala en kayakistas, sus medidas antropométricas (talla, talla sentado y envergadura) y las variables cinemáticas frecuencia de ciclo media (FCM), longitud de ciclo medio (LCM) y rendimiento en la distancia, sobre un test máximo de $500 \mathrm{~m}$. realizado por las componentes del equipo nacional español dama juvenil. Los datos cinemáticos se obtuvieron tras digitalizar las filmaciones de cada uno de los test. Este estudio sugiere que existe correlación entre la longitud de la pala (LP) y la talla de los palistas, y no entre la LP y la talla sentado o la envergadura. No existe correlación entre la LP y la FCM, pero sí se entre LP y LCM. La dureza de la pala (DP) no muestra correlación con ninguna de las variables cinemáticas estudiadas.
\end{abstract}

PALABRAS CLAVE: Piragüismo; kayak; cinemática; antropometría.

* O presente trabalho não contou com apoio financeiro de nenhuma natureza, tampouco houve conflitos de interesses para a sua realização. El presente trabajo no ha contado con apoyo financiero de ninguna naturaleza para su realización. No hubo conflictos de intereses para llevar a cabo este estudio. 


\section{INTRODUCCIÓN}

La pala de kayak ha sufrido en las últimas dos décadas una gran evolución propiciada esencialmente por la aparición de nuevos materiales más resistentes que han posibilitado diseños de pala imposibles de realizar con madera (SÁNCHEZ; MAGAZ, 1993; SABDERS; Baker, 1998). Esta evolución en los materiales de construcción ha conducido a una importante modificación del gesto técnico de paleo en kayak (SABDERS; BAKER, 1998). Antiguos diseños de pala como el modelo Liminar (basadas en el arrastre) y Húngara (basada en la frecuencia de paleo), dejaron paso a nuevas hojas fundamentadas en el principio de Bernouille (modelos Wing y Rasmussen), produciendo un cambio radical en la concepción del apoyo en el agua. Para Sabders y Baker (1998), Endicott y Biccochi (1968) y Logan y Holt (1985), estos diseños revolucionarios permitían el uso de la rotación del tronco como elemento motor y un mejor enganche de la pala en el ataque.

Según Sánchez y Magaz ( 1993), la longitud de la pala (LP) y el giro o repaleo entre hojas no son aspectos que condicionen la técnica, pero sí han de tenerse en cuenta a fin de optimizar este elemento. Marek y cols (1960) proponen los valores antropométricos del palista como la base para la configuración de las dimensiones de las palas tradicionales (modelos Liminar y Húngara). Según este autor, la LP debe configurarse a partir de distintas medidas antropométricas del tren superior (anchura de hombros, longitud del brazo, altura del hombro) y la relación entre éstas. La LP en los modelos Liminar o Húngara en piragüistas de alto nivel oscilaba entre 220 y $224 \mathrm{~cm}$ para hombres y 216 y $220 \mathrm{~cm}$ para mujeres (SÁNCHEZ; MAGAZ, 1993). Diferentes autores muestran opiniones opuestas en cuanto al ajuste o modificación necesaria para los modelos Wing y Rasmunssen. La longitud de estas palas según Endicott y Biccochi (1968) debe ser 2-4cm menor que los modelos tradicionales, mientras que Beaudou y Cezard (1987) proponen una pala 2-4cm mayor. Sumner y colaboradores (2003) estudiaron en un túnel de viento la fuerza aplicada por los tres diferentes diseños de hoja (Liminar, Wing, Rasmunssen) y determinaron que la fuerza de arrastre era similar en los tres modelos, aunque a medida que el ángulo aumentaba las palas Wing y Rasmunssen se tornaban más efectivas. Mann y Kearney ( 1980) determinaron que la fase propulsiva o fase acuática de la palada se emplea para mantener la velocidad horizontal de la piragua y que la efectividad de la palada aumenta cuando la pértiga se encuentra en posición vertical, posición que define como posición de potencia de la palada. Por otro lado, Kendal y Sanders (1992), Plagenhoef (1979), y Begon y cols. (2008) cuantificaron la duración de la fase aérea y la fase acuática o propulsiva, determinando este último que la fase acuática se ha reducido progresivamente con el paso de los años en el piragüismo de alto nivel. Al aumentar la velocidad de desplazamiento 
de la embarcación, la velocidad de la pala respecto a la piragua debe aumentar para mantener la magnitud de la fuerza aplicada. Sprigings y cols. (2006) proponen una ecuación para facilitar la personalización de las dimensiones de la hoja basada en la potencia aplicada en la palada a través de un kayakergómetro. No obstante, en la actualidad no existen estudios que relacionen las medidas antropométricas de los palistas con la longitud de la pala para estos nuevos modelos.

Por otro lado, el estudio de las variables cinemáticas en piragüismo se ha centrado en la última década en la distribución del esfuerzo en las distancias de competición (BISHOP y cols., 2002; IssURIN, 1998), y algo más sobre la frecuencia de paleo (FP) como uno de los parámetros a comparar entre el paleo en kayakergómetro y en agua (VAN SOMeren; Oliver, 2002; Barnes; Adams, 1999). Alacid et al. (2005) llevó a cabo un análisis de las variables cinemáticas del paleo y su relación con el rendimiento de los palistas, empleando una metodología validada en natación y que ha podido adaptarse al piragüismo por ser especialidades con características similares (carácter cíclico, medio de desplazamiento y velocidad de movimientos) (KESKINEN y cols, 1989; CostILl y cols., 1985; CRAIG y cols., 1985). En el presente estudio se pretende analizar las posibles relaciones existentes entre la configuración de la pala y los valores antropométricos de los palistas, así como posibles relaciones entre la configuración de la pala y las variables cinemáticas del paleo.

\section{MÉTODO}

\section{POBLACIÓN}

Participaron en el estudio las ocho palistas femeninas del equipo nacional juvenil de la Real Federación Española de Piragüismo. Los sujetos de la muestra comparten características antropométricas similares (talla: I65,0 $\pm 2,8 \mathrm{~cm}$; envergadura: $164,6 \pm 3,3 \mathrm{~cm}$; talla sentado: $127,4 \pm 2,3 \mathrm{~cm}$ ), de rendimiento (todas fueron finalistas en el Campeonato de España de Velocidad) y ninguna presentaba enfermedad alguna ni se encontraba sometida a tratamiento farmacológico en el periodo que se realizaron las pruebas.

\section{PROCEDIMIENTO}

Se efectuó la valoración antropométrica siguiendo las indicaciones del Grupo Español de Cineantropometría (Grec), a partir de la cual se determinó la composición corporal de las palistas según los cálculos propuestos por Yuhasz (1 974). Todas las mediciones fueron realizadas por un único observador. La variable talla se midió con 
un tallímetro con precisión de 0, I cm Seca (Seca, Alemania). Tanto la talla sentado como la envergadura se midieron con una cinta métrica inextensible milimetrada con precisión de 0, I cm Holtain (Holtain Ltd., Reino Unido).

Las palistas realizaron un test máximo de 500m a lo largo de una calle acotada por boyas cada $25 \mathrm{~m}$. Cada deportista empleó para la prueba su embarcación y pala habitual de entrenamiento y competición. Las pruebas se desarrollaron en una lámina de agua sin corrientes y en ausencia de viento que pudiese alterar los resultados. Durante la prueba se obtuvieron los valores de frecuencia cardiaca cada cinco segundos mediante un pulsómetro telemétrico Polar S6I0 (Polar, Finlandia) y los valores de concentración de lactato en sangre mediante un analizador Lactate Pro LT (Arkay Inc, Kyoto, Japón) en los minutos I, 3, 5 y 7 tras finalizar la prueba. Todos los test de agua fueron grabados con una videocámara Canon V400e (Canon Inc, Japón) desde una perspectiva lateral al palista, encuadrando la proa del kayak al paso por las boyas de salida y llegada, elevando de esta forma la precisión y fiabilidad de las mediciones y facilitando el proceso de obtención de resultados. Las grabaciones fueron digitalizadas empleando una tarjeta capturadora AverMedia TV Studio (AverMedia Tecnologies Inc, EEUU) y los datos obtenidos se registraron a través del software VirtualDub (I .4.7 by Avery Lee) y una hoja de cálculo Microsoft Excel 2000 (Microsoft Corporation, EEUU). La velocidad media (VM) se determinó a partir del tiempo total que empleó cada palista para recorrer los 500 m del test, expresando los resultados en $\mathrm{m} \cdot \mathrm{s}^{-1}$. Para establecer la frecuencia de ciclo media (FCM) se cuantificó el número total de ciclos que realizó cada palista en la prueba y se dividió por el tiempo que empleó en completar la distancia, expresando los resultados en ciclos $\cdot \mathrm{min}^{-1}$. Para obtener una mayor precisión en las mediciones se emplearon las imágenes digitalizadas y el programa VirtualDub para el análisis de los fotogramas. La longitud de ciclo media (LCM) se calculó tras dividir la VM entre la FCM obtenidas para cada palista, expresando los resultados en $\mathrm{m}$ · ciclo-1. La longitud de la pala (LP) se determinó con una cinta métrica, midiendo la distancia entre los puntos más alejados de ambas cucharas. La superficie de la hoja se determinó plasmando sobre una superficie plana el área de la misma. Una vez obtenido el perímetro de la cuchara en dos dimensiones, se introdujeron en el programa AutoCAD 2005 (Autodesk, EEUU) las coordenadas ( $x, y$ ) de los puntos que conformaban dicho perímetro (Fig. 3).

Para este estudio se introdujo el concepto de dureza de la pala (DP), que relaciona los valores de la LP y la superficie total de empuje con objeto de mostrar un valor que represente la exigencia física que requiere una determinada configuración de pala. Esta variable resultó de la suma de las áreas de las hojas y su producto con la longitud de la pala, expresando los resultados en $\mathrm{cm}^{3}$. Durante todo este protocolo 


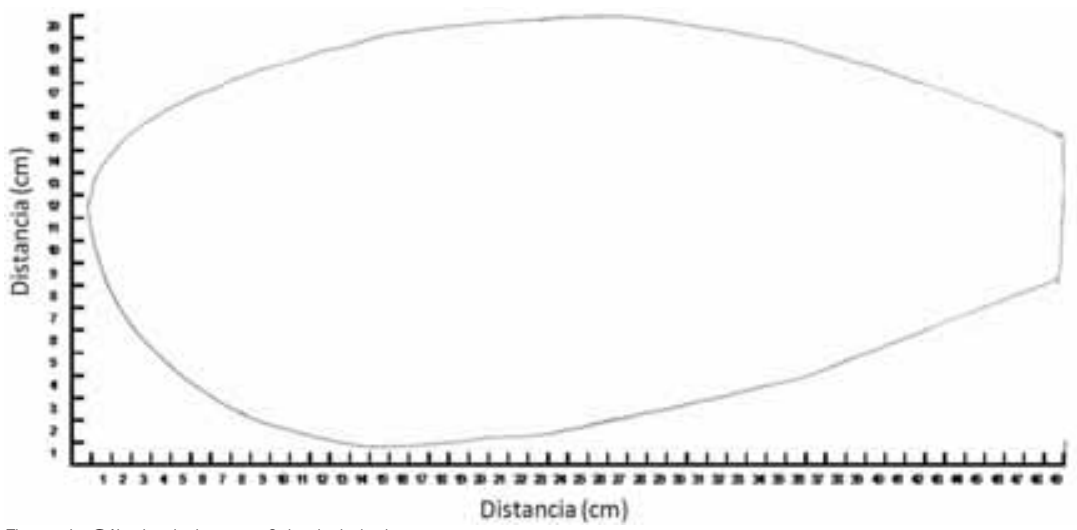

Figura I: Cálculo de la superficie de la hoja.

se confirma que el tipo de observación planteada es directa y no participante, lo que ha llevado a necesitar, para conseguir registrar los datos con suficiente fiabilidad, un riguroso entrenamiento de observadores.

En cuanto al análisis estadístico de los datos, se utilizó el paquete informático SPSS 12.0. La relación entre las variables analizadas se realizó a través de un análisis de regresión lineal y del cálculo del coeficiente de correlación de Pearson. En todo caso se estableció un intervalo de confianza del 95\%.

\section{RESULTADOS}

Los datos obtenidos para cada sujeto de los registros de frecuencia cardiaca máxima, concentración de lactato máximo, así como los valores de la dimensión de la pala se muestran en la Tabla I.

Tabla I: Registros de frecuencia cardiaca, concentración de lactato y dimensiones de la pala

\begin{tabular}{llllll}
\hline & $\mathrm{FC}_{\text {max }}(\text { lat } \cdot \text { min })^{-1}$ & {$[\text { Lact }]_{\text {máx. }}\left(\mathrm{mMol} \cdot \mathrm{l}^{-1}\right)$} & $\begin{array}{l}\text { Longitud de la } \\
\text { pala }(\mathrm{cm})\end{array}$ & $\begin{array}{l}\text { Superficie de } \\
\text { la hoja }\left(\mathrm{cm}^{2}\right)\end{array}$ & $\begin{array}{l}\text { Dureza de la pala } \\
\left(\mathrm{cm}^{3} \cdot 10000^{-1}\right)\end{array}$ \\
\hline Media & 186.8 & 12.6 & 207.9 & 700.8 & 29.1 \\
DE & 7.0 & 1.2 & 1.9 & 18.1 & 0.9 \\
\hline
\end{tabular}

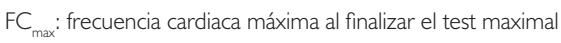

$[\text { Lact }]_{\text {máx: }}$ : concentración máxima de ácido láctico al finalizar el test maximal. 
La Figura 2 muestra las correlaciones entre la longitud de la pala y los valores antropométricos de las palistas.
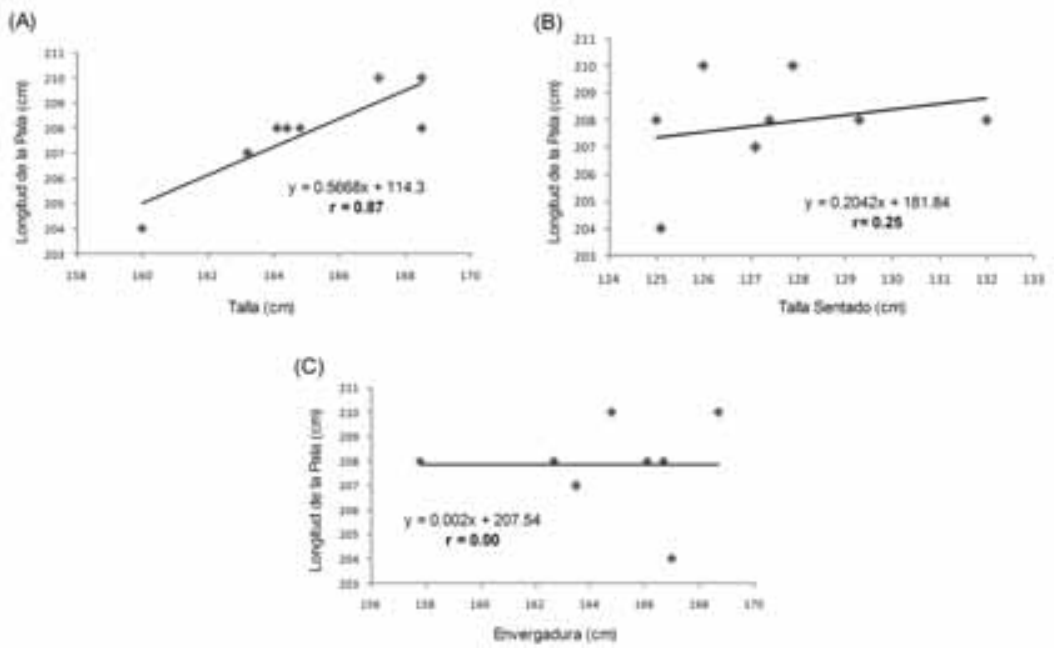

Figura 2: Correlaciones entre la longitud de la pala y las variables antropométricas de talla (A), talla sentado (B) y envergadura $(C)$.

La Figura 3 muestra las correlaciones entre las distintas variables que configuran las dimensiones de la pala y las variables cinemáticas de paleo.
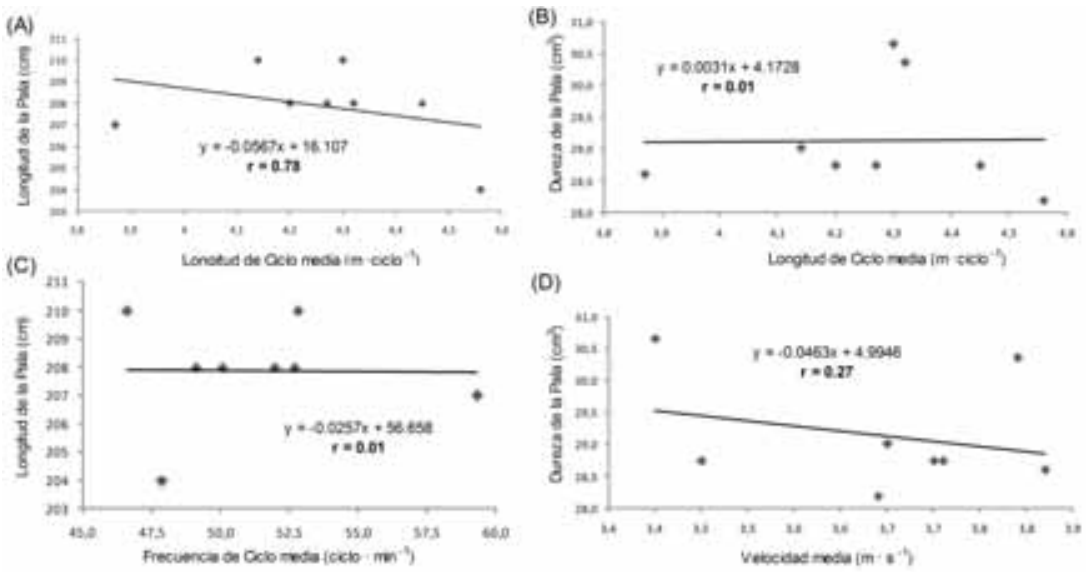

(D)

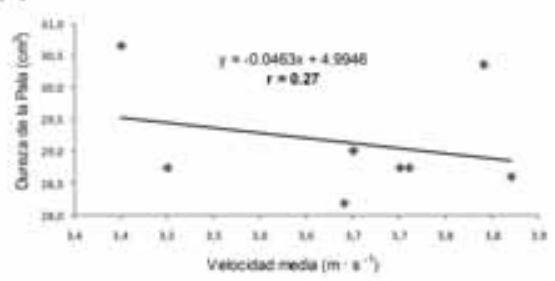

Figura 3: Correlación entre las dimensiones de la pala y las variables cinemáticas Frecuencia de ciclo media, Longitud de ciclo media y Velocidad media. 


\section{DISCUSIÓN}

De los resultados obtenidos de las correlaciones entre la LP y las distintas variables antropométricas, destaca la alta correlación existente entre la configuración de la pala y la talla de los palistas (Fig. 2A), es decir, los palistas más altos emplean palas más largas. Por otro lado sorprende que las correlaciones obtenidas para otras variables antropométricas como la talla sentado y la envergadura, muestren valores de correlación tan bajos (Fig. 2B y 2C). Estas relaciones parecen indicar que palistas y técnicos configuran las dimensiones de la pala por el método de ensayo error, buscando la comodidad del palista, o a lo sumo tomando como única referencia objetiva la talla del sujeto (Fig. 2). Teniendo en cuenta la posición de base que ha de adoptar el kayakista en la piragua (SABDERS; Baker, I998; MAnN; KeARney, 1980; SANDERS; KendAL, 1992) (Fig. 4), si el único criterio objetivo que se está empleando para determinar la longitud de la pala es la talla del palista, cabe la posibilidad de que se estén cometiendo errores en la configuración. Podría parecer más adecuado utilizar los valores de la talla sentado y la envergadura para determinar la longitud de la pala. Si sólo se tiene en cuenta la talla, podríamos encontrar palistas con piernas muy largas en relación a su talla sentado (medida antropométrica que determina la posición de paleo) o palistas con poca envergadura en relación a su talla (valor relacionado con el agarre y aplicación de fuerzas sobre la pértiga), por lo que el ajuste de la pala sería erróneo.

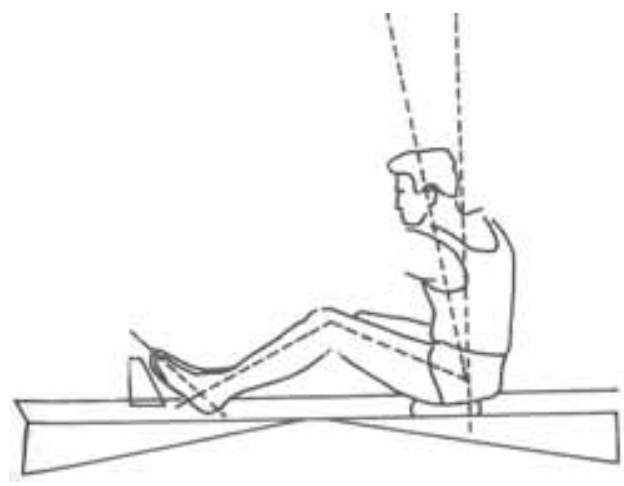

Figura 4: Posición base del kayakista (Modificado de Sánchez y Magaz, 1993).

En el estudio de las correlaciones entre las distintas variables que configuran las dimensiones de la pala y las variables cinemáticas, destaca una alta correlación entre la LP y la LCM (Fig. 3A). De dicha relación se puede intuir que a mayor longitud de 
la pala, mayor será el recorrido de ésta en la fase acuática, y por consiguiente mayor avance de la piragua por ciclo de paleo. Estos datos parecen confirmarse en parte por las aportaciones del estudio de Alacid y cols. (2005), autores que detectaron bajos niveles de correlación entre la LCM y la FCM con la VM en piragüistas infantiles.

Por otro lado, la DP no muestra correlación alguna con la LCM (Fig. 3B), seguramente debido a que la superficie de empuje de las hojas no esta directamente relacionada con valores antropométricos, y sí podría estarlo con otros factores como la condición física del palista y más concretamente con los niveles de fuerza que desarrolle (SABDERS; BAKER, 1998; LogAN; HOLT, 1985). En el análisis de la relación entre la FCM y la LP (Fig. 3C) sorprende la ausencia de correlación entre ambas. Estos datos no coinciden con los hallados por Alacid y cols. (2005) en piragüismo ni por otros autores en natación (Pal y cols., 1984; KenNedy y cols., 1990; CHENGALUR; BROWN, 1992; ArELLANO y cols., 1994) que si encontraron correlaciones negativas entre la FCM y LCM. Además, Sánchez y Magaz (1993) encontraron una correlación negativa entre la LP y la FCM, datos que no se confirman en este estudio. Finalmente, la Figura 3D muestra la ausencia de correlación entre la DP y la VM. Este hecho indica la multitud de factores que afectan al rendimiento global en el piragüismo (factores físicos, psíquicos, técnicos y tácticos) (SánCHEZ; MAGAZ, 1993; BISHOPy cols., 2002; ISSURIN, 1998; SANDERS; KendAL, 1992; SánCHEZ, 1993), no pudiendo simplificar el rendimiento final del palista al parámetro de la dureza de la pala.

\section{CONCLUSIONES}

Se observan altos valores de correlación entre la longitud de la pala y la talla de los palistas y por el contrario no se encuentran relaciones directas entre la longitud de la pala y otras variables antropométricas como son la talla sentado y la envergadura, que muestran bajos valores de correlación. Este estudio muestra por tanto como la configuración de la pala se está realizando en base a medidas antropométricas sin relación alguna con la posición de base del kayakista como la talla, y no en función de otras medidas antropométricas con mayor relación con esta posición de base como es la talla sentado y la envergadura.

No existe correlación alguna entre la longitud de la pala y la frecuencia de ciclo, aunque si existe correlaciones aceptables entre las variables de longitud de la pala y la longitud de ciclo.

La dureza de la pala no muestra correlación con ninguna de las variables cinemáticas estudiadas, hecho que demuestra el gran número de variables que condicionan el éxito del piragüista en competición. 
Configuração dos remos de caiaquistas, suas medidas antropométricas e as variáveis cinemáticas

RESUMO: Foram analisadas as correlações entre a configuração dos remos de caiaquistas, suas medidas antropométricas (altura, altura sentado e envergadura) e as variáveis cinemáticas, frequência de ciclo média (FCM), comprimento do ciclo médio (LCM) e rendimento em distância, a partir de um teste máximo de $500 \mathrm{~m}$, realizado pelas componentes da equipe nacional espanhola juvenil feminina. Os dados cinemáticos foram obtidos pela digitalização das filmagens de cada um dos testes. Do estudo se depreende que existe correlação entre o comprimento do remo $(L P)$ e o tamanho dos remadores, e não entre a $L P$, porém entre o tamanho sentado e a envergadura. Não existe correlação entre a LP e a FCM, mas sim entre a LP e a LCM. A exigência física requerida do caiaquista (DP) não mostra correlação com nenhuma das variáveis cinemáticas estudadas.

PALAVRAS-CHAVE: Caiaquismo; caiaque; cinemática; antropometria.

\section{Configuration of the kayak paddle, anthropometric measurements and kinematc parameters}

ABSTRACT: We examined the correlations between the configuration of the kayak paddle, anthropometric measurements of the kayakers (height, sitting height and arm span) and the kinematic variables average frequency cycle (FCM), average length of cycle (LCM) and performance in the distance, about $500 \mathrm{~m}$. test in the components of the female Spanish Junior National Team. Kinematic variables were obtained after digitizing the video test. The study shows that there is correlation between the length of the paddle (LP) and the height of paddlers, and not between the LP and other anthropometric variables such as sitting height and wingspan. There is no correlation between the LP and FCM, but there are between LP and LCM. The hardness of the paddle (DP) shows no correlation with any of the kinematic variables studied.

KEY WORDS: Canoeing; kayak; kinematics; anthropometric.

\section{REFERENCIAS}

ALACID, F.; FERRER, V.; MARTÍNEZ, E.; CARRASCO, L. Análisis cuantitativo de la técnica de paleo en kayakistas infantiles. Motricidad. European Journal of Human Movement, Madrid, n. 13, p. 133-|46, 2005.

ARELLANO, R.; BROWN, P.; CAPPAERT, J.; NELSON, R. C. Analysis of 50, 100 and 200m freestyle swimmers at the 1992 Olympic Games. Journal of Applied Biomechanics, Champaign, v. 10, n. 2, p. 189-199, 1994.

BARNES, C. A; ADAMS, P. C. Realiability and criterion validity of a 120 s maximal sprint on a kayak ergometer. Journal of Sports Science, London, v. 16, n. I, p. 25-26, 1999.

BEAUDOU, A; CEZARD, J. Practique du canoe-kayak. Paris: Editorial Vogot, 1987. 
BEGON, M.; LACOUTURE, P.; COLLOUD, F. 3D kinematic comparison between onwater and on ergometer. In: KAYAKING ISBS CONFERENCE 2008, Seoul, Korea, July 14-18, 2008.

BISHOP, D.; BONETTI, D.; DAWSON, B. The influence of pacing strategy on VO2 max and supramaximal kayak performance. Medicine and Science in Sport and Exercise, v. 34, n. 6 , p. 1041 - 1047, 2002.

CHENGALUR, S. N.; BROWN, P. L. An analysis of male and female Olympic swimmers in the 200-meter events. Canadian Journal of Sport Sciences, v. 17, n. 2, p. 104-109, 1992.

COSTILL, D. L.; KOVALESKI, J.; PORTER, D.; FIELDING, R; KING, D. Energy expendidure during front crawl swimming: prediction in middle distance events. International Journal of Sport Medicine, v. 6, n. 5, p. 266-270, 1985.

CRAIG, A. B.; SKEHAN, P. L.; PAWELCZYK, J. A.; BOOMER, W. L. Velocity, stroke rate, and distance per stroke during elite swimming competition. Medicine and Science in Sports and Exercise, v. 17, n. 6, p. 625-634, 1985.

ENDICOTT, W; BICCOCHI, B. Interview du concepter de la pagaie Wing. Cahier Technique, n. 4, p. $281-194,1968$.

ESPARZA, F. Manual de cineantropometría. Pamplona: FEMEDE, 1993.

ISSURIN, V. Analysis of the race strategy of world-class kayakers. En: ISSURIN, V. Science \& practice of canoe/kayak high-performance training: selected articles in memory of junior world champion Nevo Eitan. Tel Aviv: Elite Sport Departament of Israel, p. 27-39, 1998.

KENDAL, S.; SANDERS, R. The technique of elite flatwater kayak paddlers using the wing paddle. International Journal of Sport Biomechanics, Champaign, v. 8, n. 3, p. 233-250, 1992.

KENNEDY, P.; BROWN, P.; CHENGALUR, S. N; NELSON, R. C. Analysis of male and female Olympic swimmers in the 100-meter events. International Journal of Sport Biomechanics, Champaign, v. 6, n. 2, p. 187-197, 1990.

KESKINEN, K; TILLI, L. J.; KOMI, P. Maximum velocity swimming: interrelationships of stroking characteristics, force production and anthropometric variables. Scandinavian Journal of Sport Sciences, Helsinki, v. II, n. 2, p. 87-92, 1989.

LOGAN, M.; HOLT. E. The flatwater kayak stroke. National Stregth and Conditioning Asociation Journal, Colorado Springs, v. 5, n. 7, p. 4-। I, 1985.

MANN, R.; KEARNEY, J. A biomechanical analysis of Olimpic-style flatwater kayak stroke. Medicine and Science in Sport and Exercise, Philadelphia, v. 12, n. 3, p. I83-188. 1980. 
MAREK, L.; DVORAK, B.; ALBERT, L. Technica du canoe. Cahier Technique, Paris, v. 4, n. 27, p. 10-32, 1960.

PAI, Y. C.; HAY, J. G; WILSON, B. D. Stroking techniques of elite swimmers. Journal of Sport Sciences, London, v. 2, n. 3, p. 225-239, 1984.

PLAGENHOEF, S. Biomechanical analysis of Olympic flatwater kayaking and canoeing. Research Quaterly, Berlin, v. 50, n. 3, p. 443-459, 1979.

SABDERS, H.; BAKER, D. Evolution of technique in flatwater kayaking. En: ISSURIN, V. Science \& practice of canoe/kayak high-performance training: selected articles in memory of junior world champion Nevo Eitan. Tel Aviv: Elite Sport Departament of Israel, p. 67-81, 1998.

SÁNCHEZ, J; MAGAZ, S. La técnica. En: SÁNCHEZ J. L. Piragüismo (I). Madrid: COE, p. $101-386,1993$.

SÁNCHEZ, M. Acondicionamiento físico en seco. En: SÁNCHEZ, M. Piragüismo (II). Madrid: COE, p. 20-101, 1993.

SANDERS, R.; KENDAL, S. A description of Olympic flatwater kayak stroke technique. Australian Journal of Science and Medicine in Sport, Melbourne, v. 24, n. I, p. 25-30, 1992.

SPRIGINGS, E.; MCNAIR, P.; MAWSTON, G.; SUMNER, D.; BOOCOCK, M. A method for personalising the blade size for competitors in flatwater kayaking. Sports Engineering, London, v. 9, n. 3, p. 147-153, 2006.

SUMNER, D.; SPRIGINGS, E. J.; BUGG, J. D.; HESELTINE, J. L. Fluid forces on kayak paddle blades of different design. Sports Engineering, London, v. 6, n. I, p. I I-20, 2003.

VAN SOMEREN, K. A.; OLIVER, J. E. The efficacy of erometry determinated herat rates for flatwater kayak training. International Journal Sport Medicine, Stuttgart, v. 23, n. I, p. 28-32, 2002.

YUHASZ, M. S. Physical fitness manual. Ontario: University of Western Ontario, 1974.

Recebido: 10 jan. 2009

Aprovado: 15 dez. 2009

Endereço para correspondência

Dr. Arturo Díaz Suárez

C/ Argentina s/n Santiago de la Ribera

30720 Murcia (España) 